\title{
Cáncer de próstata
}

A. Zapatero

A lo largo de las dos últimas décadas han emergido dos nuevos conceptos encaminados a mejorar los resultados en los pacientes con cáncer de próstata localizado, fundamentalmente la intensificación o escalada de dosis con nuevas tecnologías de radioterapia, como la radioterapia conformacional 3D (RTC-3D) con o sin intensidad modulada (IMRT) o la braquiterapia transperineal 3D y por otro, el empleo combinado de radioterapia y supresión androgénica'.

\section{Radioterapia conformacional 3D e intensificación de dosis en cáncer de próstata localizado}

La evidencia publicada procedente de estudios retrospectivos, estudios prospectivos fase I y II tanto mono-institucionales como de grupos cooperativos y finalmente de ensayos aleatorizados fase III, parece haber confirmado las hipótesis de trabajo desarrolladas a finales de los ochenta con la nueva tecnología de radioterapia conformacional tridimensional (RTC3D) ${ }^{2-7}$. A saber: 1) Que la RTC3D comparada con RT convencional permite disminuir de forma significativa la morbilidad derivada; 2) Que existe una relación dosis respuesta en el cáncer de próstata; 3) Que es posible y seguro incrementar la dosis de radiación en el volumen tumoral sin aumentar la toxicidad en tejidos sanos; 4) Que este aumento de la dosis ha conseguido un aumento significativo en el control bioquímico y control local, y en última instancia un aumento probable de la supervivencia.

Este efecto (dosis-respuesta) ampliamente documentado en los ensayos de intensificación de dosis en cáncer de próstata, ha resultado más evidente en pacientes considerados de "riesgo intermedio". En los pacientes con enfermedad de bajo riesgo, todavía existe controversia sobre si benefician o no de dosis altas de radiación, si bien datos procedentes de estudios monoinstitucionales (MSKCC) como cooperativos

Servicio de Oncología Radioterápica Hospital Universitario de La Princesa Madrid (ref de D'Amico (ASTRO 2003) parecen confirmar una dosis respuesta 8,9 . Finalmente, los pacientes de alto riesgo, con potencial mortalidad derivada de enfermedad micrometastásica oculta al diagnóstico, aunque parecen beneficiarse localmente de un aumento de la dosis de radiación, su impacto en supervivencia es aún limitado. En este sentido, hay que tener en cuenta que puede resultar muy difícil separar los datos de la enfermedad localmente avanzada de una población muy extensa de pacientes con cáncer de próstata con al menos uno de los factores considerados de alto riesgo (PSA $>20 \mathrm{ng} / \mathrm{ml}$, o Gleason > 7). Los ensayos actuales en marcha, como el RTOG P-0126, servirán no sólo de validación de la evidencia actual, sino que proporcionarán información adicional sobre toxicidad, resultados histopatológicos y de calidad de vida.

\section{Supresión androgénica en combinación con radioterapia}

La justificación para combinar la supresión androgénica con la radioterapia radical procede de datos experimentales y de la práctica clínica. Es bien conocido que la ablación androgénica produce disminución del volumen tumoral (citorreducción). Esta reducción resultaría en una disminución de la hipoxia tumoral, aumentando por tanto la radiosensibilidad. Asociado, está descrito un enlentecimiento del ciclo celular mediado por el bloqueo androgénico que implicaría una disminución de la repoblación celular durante la irradiación. Estudios recientes indican que la deprivación androgénica puede producir muerte celular por apoptosis. Sobre esta base se ha especulado que la administración concurrente o secuencial de supresión androgénica y radioterapia podría tener un efecto aditivo (confirmado en modelos animales) $\mathrm{o}$ inclusive supraaditivo ${ }^{10,11,12}$. Finalmente, la hormonoterapia administrada de forma adyuvante a radioterapia actuaría esterilizando las micrometástasis ocultas, mientras que la irradiación mataría las células tumorales independientemente de su hormonosensibilidad en un fenómeno de cooperación espacial similar al que ocurre con la radioquimioterapia.

Pero la cuestión importante es: ¿existe realmente un beneficio clínico para los pacientes con la asociación de hormonoterapia y radioterapia? En la actualidad, disponemos ya de los resultados maduros de múltiples estudios aleatorizados mono 


\section{A. Zapatero}

TABLA I

Resumen ensayos Fase III de deprivación androgénica en combinación con radioterapia externa dosis convencionales

\begin{tabular}{|c|c|c|c|c|c|}
\hline Serie & $N$ & Estadio & Secuencia HT & SLE/CL & SG \\
\hline QUEBEC & $\begin{array}{l}41 \\
43 \\
36\end{array}$ & B1-T3C & $\begin{array}{l}\text { RT } \\
\text { BAM pre+RT } \\
\text { BAM pre+RT+6m }\end{array}$ & $\begin{array}{l}66.7(B Q) \\
72.4 \\
84.6\end{array}$ & - \\
\hline RTOG & 230 & T2C-T4 N+ & RT & $/ 29$ & 60 \\
\hline 8610 & 226 & & $\begin{array}{l}\text { BAM pre+RT } \\
\text { Update } 2001\end{array}$ & $\begin{array}{l}/ 54 \\
\uparrow S G \text { Gl 2-6? }\end{array}$ & 60 \\
\hline Granfors & $\begin{array}{l}46 \\
45\end{array}$ & $\begin{array}{l}\mathrm{T} 1-4 \mathrm{NO} 0-3 \\
\mathrm{~N}+>S G\end{array}$ & Castración $\infty$ & $\begin{array}{l}73 \\
56\end{array}$ & \\
\hline Zagars & $\begin{array}{l}38 \\
40\end{array}$ & T3 & $\mathrm{DES} \infty$ & $\begin{array}{l}71 / \\
49 /\end{array}$ & $\begin{array}{l}68 \\
73\end{array}$ \\
\hline $\begin{array}{l}\text { EORTC } \\
22863\end{array}$ & $\begin{array}{l}195 \\
196\end{array}$ & TIAG-T4 & GOS RT + 3años & $\begin{array}{l}74 / 97 \\
40 / 77\end{array}$ & $\begin{array}{l}78 \\
62 \\
p=.0002\end{array}$ \\
\hline $\begin{array}{l}\text { RTOG } \\
8531\end{array}$ & $\begin{array}{l}477 \\
468\end{array}$ & $\begin{array}{l}\text { T1-2N1T3 } \\
\text { Gleason 8-10: }\end{array}$ & $\begin{array}{l}\text { GOS RT }+\infty \\
>S G p=.036\end{array}$ & $\begin{array}{l}84 / 60 \\
71 / 44\end{array}$ & $\begin{array}{l}75 \\
71\end{array}$ \\
\hline $\begin{array}{l}\text { RTOG } \\
92-02\end{array}$ & 1554 & $\begin{array}{l}\text { T2C-T4 } \\
\text { Gleason(8 }\end{array}$ & $\begin{array}{l}\text { BAM preRT+2años } \\
\text { preRT+2años }\end{array}$ & $\begin{array}{l}54 \text { SLP } \\
34\end{array}$ & $\begin{array}{l}78 \\
79 \\
80\end{array}$ \\
\hline $\begin{array}{l}\text { RTOG } \\
93-14\end{array}$ & 1323 & $\begin{array}{l}\text { Riesgo N+ } \\
>15 \%\end{array}$ & $\begin{array}{l}\text { RT+/- pelvis y } \\
\text { HT } 4 \mathrm{~m} \text { neo vs } 4+4 \mathrm{~m}\end{array}$ & $\begin{array}{l}56 \% \text { vs } 46 \% \\
53 \% \text { vs } 48 \%\end{array}$ & \\
\hline
\end{tabular}

TABLA ॥

Resultados de estudios publicados de RTC-3D en intensificación de dosis asociada con deprivación androgénita neoadyuvante

\begin{tabular}{|c|c|c|c|c|}
\hline Autor & $\mathbf{N}$ & По० & SLFB/SG & Morbilidad \\
\hline $\begin{array}{l}\text { Martínez } \\
\text { (J Urol 2003) }\end{array}$ & 507 & $\begin{array}{l}\text { RTE+HDR } \\
\pm 6 \mathrm{~m} \mathrm{HT}\end{array}$ & $\begin{array}{l}76 \% / 87 \% \text { NS } \\
74 \% / 81 \%\end{array}$ & Impotencia \\
\hline $\begin{array}{l}\text { Kupelian } \\
\text { (Cancer 2004) }\end{array}$ & 1352 & $\mathrm{RT}-\mathrm{ID} \pm \mathrm{HT}$ & $\begin{array}{l}\text { AMV: HT-NS } \\
\text { AMV: Dosis RT Sig }\end{array}$ & - \\
\hline $\begin{array}{l}\text { Nguyen } \\
\text { (IJROBP 2003) } \\
\text { Jani } \\
\text { (AmJClinOncol) }\end{array}$ & $\begin{array}{l}296 \\
\text { high risk } \\
412 \\
\text { todos }\end{array}$ & $\begin{array}{l}\text { RT-ID } \pm \mathrm{HT} \\
\sim 3 \mathrm{~m} \\
\text { RT-66-72 } \\
\pm \mathrm{HT} 4 \mathrm{~m}\end{array}$ & $\begin{array}{l}\text { AMV: HT-NS } \\
\text { AMV: Dosis RT Sig } \\
83 \% \text { vs } 73 \% ; p=0.02\end{array}$ & Sangr. Rectal \\
\hline $\begin{array}{l}\text { Valicenti } \\
\text { (IJROBP 2003) }\end{array}$ & 583 & $\begin{array}{l}\text { RT-ID } 9406 \\
\pm 6-9 \mathrm{~m} \mathrm{HT}\end{array}$ & $\begin{array}{l}\text { AMV: HT-NS } \\
\text { AMV: Dosis RT Sig }\end{array}$ & Urinaria? \\
\hline $\begin{array}{l}\text { Geinitz } \\
\text { (SR 2002) }\end{array}$ & 180 & $\mathrm{RT}-\mathrm{ID} \pm \mathrm{HT}$ & AMV: HT-NS & - \\
\hline $\begin{array}{l}\text { Zapatero } \\
\text { (Radiother Oncol 2002) }\end{array}$ & 150 & $\mathrm{RT}-\mathrm{ID} \pm \mathrm{HT}$ & AMV: HT-NS & - \\
\hline $\begin{array}{l}\text { GICOR } \\
\text { (NP) }\end{array}$ & 396 & $\mathrm{RT}-\mathrm{ID} \pm \mathrm{HT}$ & & \\
\hline
\end{tabular}

y multi-insitucionales, con más de 4.000 pacientes reclutados, que han estudiado el papel de la combinación de hormonoradioterapia en el cáncer de próstata localizado ${ }^{13-15}$.

Existen al menos 9 ensayos prospectivos aleatorizados que han investigado el beneficio potencial de la asociación de deprivación androgénica (mayoritariamente con goserelina) bien neoadyuvante y de corta duración (habitualmente menos de 6 meses), bien adyuvante y generalmente prolon- gada (entre dos años e indefinida) con radioterapia externa radical en pacientes con cáncer de próstata localizado ${ }^{13-21}$ (Tabla I).

Todos estos ensayos (muchos de ellos pre-PSA) han empleado RT a dosis convencional entre 65.0 y $70 \mathrm{~Gy}$. Generalmente, el volumen de irradiación incluía de alguna forma y en una primera fase cadenas ganglionares pélvicas (45.0$50.0 \mathrm{~Gy}$ ). Los criterios de inclusión eran extensos (T1-T4, 
NO-pN+, cuaquier grado Gleason) y no se estratificaba por subgrupos de riesgo. Finalmente, la gran mayoría tampoco incluía al diagnóstico análisis por PSA.

De estos 9 ensayos la mayoría han confirmado que la combinación de radioterapia y supresión androgénica se asocia a un beneficio significativo en control bioquímico, loco-regional y en la supervivencia libre de metástasis. Pero sólo el ensayo de Bolla ha demostrado beneficio significativo en la supervivencia global y causa-específica en toda su serie. De forma adicional, tanto la actualización de los ensayos RTOG 85-31 y RTOG 92-0, como su metaanálisis, confirman este impacto positivo en supervivencia con la adición de supresión androgénica prolongada en subgrupos desfavorables con Gleason 8-10.

Las potenciales conclusiones derivadas de estos ensayos de deprivación androgénica en combinación con radioterapia convencional, parecen confirmar un beneficio en supervivencia global para aquellos pacientes con enfermedad del alto riesgo (T3-4 y Gleason 8-10) cuando se utilizan esquemas de hormonoterapia prolongada ( $\geq 2$ años), y posiblemente para pacientes con T3 y Gleason $\leq 6$ con hormonoterapia corta neoadyuvante.

\section{3. ¿Cuál es el papel de la deprivación androgénica en combinación con radioterapia 3D en intensificación de dosis?}

Debido a que los ensayos aleatorizados fase III que han demostrado beneficio con el empleo de DA (Tabla II) han empleado de forma exclusiva dosis de radiación convencionales entre 65.0 Gy y 70.0 Gy, la cuestión que se deriva a continuación, de si el empleo de DA podría conducir a un beneficio adicional en pacientes tratados con RTC-3D a dosis altas, está sin resolver. En la actualidad no existe ningún ensayo aleatorizado publicado en este sentido. Aunque diversos autores han presentado resultados de estudios prospectivos y retrospectivos mono-institucionales tratando de contestar esta controversia, ${ }^{22-24}$ los datos han sido contradictorios.

Gran parte de estos estudios han empleado cursos cortos de supresión androgénica (neoadyuvante y concomitante) en general menor de 6 meses. Mientras que la mayoría no han encontrado beneficio significativo en control bioquímico con esta asociación hormono-radioterapia, algunos han publicado incluso una mayor incidencia de complicaciones tardías con la combinación de tratamientos.

Las cuestiones que surgen a continuación son: a) si la supresión androgénica podría sustituir la intensificación de dosis en los pacientes de riesgo y b) si existe una potenciación de la morbilidad aguda o tardía derivada del empleo de la combinación de tratamientos. Confiamos que estas y otras cuestiones serán contestadas en los ensayos aleatorizados que se encuentran en marcha.

\section{Bibliografía}

1. Zapatero A, Pérez Torrubia A. Cáncer de Próstata Localizado: ¿Existe un Papel para la Deprivación Androgénica Asociada a Radioterapia? Rev Oncología 1999; 1:175-80.

2. Pollack A, Zagars GK, Starkschall G, Antolak JA, Lee JJ, Huang $E$, et al. Prostate cancer radiation dose response: results of the M. D. Anderson phase III randomized trial. Int J Radiat Oncol Biol Phys 2002; 53(5): 1097-105.

3. Zelefsky MJ, Leibel SA, Gaudin PB, Kutcher GJ, Fleshner NE, Venkatramen ES, et al. Dose escalation with three-dimensional conformal radiation therapy affects the outcome in prostate cancer. Int J Radiat Oncol Biol Phys 1998; 41(3):491-500.

4. Zelefsky MJ, Fuks Z, Hunt M, Lee HJ, Lombardi D, Ling CC, et al. High dose radiation delivered by intensity modulated conformal radiotherapy improves the outcome of localized prostate cancer. J Urol 2001;166(3):876-81.

5. Fiveash JB, Hanks $G$, Roach $M$, Wang $S$, Vigneault $E$, McLaughlin PW, ert al. 3D conformal radiation therapy (3DCRT) for high grade prostate cancer: a multi-institutional review. Int J Radiat Oncol Biol Phys 2000; 47(2):335-42.

6. Vicini FA, Abner A, Baglan KL, Kestin LL, Martínez AA. Defining a dose-response relationship with radiotherapy for prostate cancer: is more really better? Int J Radiat Oncol Biol Phys $2001 ; 51(5): 1200-8$

7. Hanks GE, Hanlon AL, Epstein B, Horwitz EM. Dose response in prostate cancer with 8-12 years' follow-up. Int J Radiat Oncol Biol Phys 2002; 54(2):427-35.

8. Leibel SA, Fuks Z, Zelefsky MJ, Hunt M, Burman CM, Mageras GS, et al. Technological advances in external-beam radiation therapy for the treatment of localized prostate cancer. Semin Oncol. 2003; 30(5):596-615.

9. Kupelian PA, Kuban D, Thames H, Levy E, Horwitz E, Martínez $A$, et al. Improved biochemical relapse-free survival with increased external radiation doses in patients with localized prostate cancer: the combined experience of nine institutions in patients treated in 1994 and 1995 (Abstr.). Int J Radiat Oncol Biol Phys. 2003; 57(2 Suppl):S271-S272.

10. Zietman AL, Prince EA, Nakfoor BM, Shipley WU. Neoadjuvant androgen suppression with radiation in the management of locally advanced adenocarcinoma of the prostate: experimental and clinical results. Urology. 1997 Mar;49(3A Suppl):74-83.

11. Pollack A, Ashoori F, Sikes $C$, et al. The early supra-additive apoptotic response of R3327-G prostate tumors to androgen ablation and radiation is not sustained with multiple fractions. Int J Radiat Oncol Biol Phys. 2000 Jan 1; 46(1):153-8.

12. Joon DL, Hasegawa M, Sikes $C$, et al A. Supraadditive apoptotic response of R3327-G rat prostate tumors to androgen ablation and radiation. Int J Radiat Oncol Biol Phys. 1997 Jul 15; 38(5):1071-7.

13. Bolla $M$, Collette $L$, Blank $L$, et al. Long-term results with immediate androgen suppression and external irradiation in patients with locally advanced prostate cancer (an EORTC study): a phase III randomized trial. Lancet 2002; 360(9327):103-6.

14. Pilepich MV, Winter K, John MJ, et al. Phase III radiation therapy oncology group (RTOG) trial 86-10 of androgen deprivation adjuvant to definitive radiotherapy in locally advanced carcinoma of the prostate. Int J Radiat Oncol Biol Phys 2001; 50(5): 1243-52.

15. Lawton CA, Winter K, Murray K, et al. Updated results of the phase III Radiation Therapy Oncology Group (RTOG) trial 85- 


\section{A. Zapatero}

31 evaluating the potential benefit of androgen suppression following standard radiation therapy for unfavorable prognosis carcinoma of the prostate. Int J Radiat Oncol Biol Phys 2001; 49(4):937-46

16. Granfors T, Modig H, Damber JE, Tomic R. Combined orchiectomy and external radiotherapy versus radiotherapy alone for nonmetastatic prostate cancer with or without pelvic lymph node involvement: a prospective randomized study. J Urol 1998 Jun; 159(6):2030-4.

17. Zagars GK, Johnson DE, von Eschenbach AC, Hussey DH. Adjuvant estrogen following radiation therapy for stage $C$ adenocarcinoma of the prostate: long-term results of a prospective randomized study. Int J Radiat Oncol Biol Phys 1988 Jun; 14(6):1085-91.

18. The Medical Research Council Prostate Cancer Working Party Investigators Group. Immediate versus deferred treatment for advanced prostatic cancer: initial results of the Medical Research Council Trial. Br J Urol 1997 Feb; 79(2):235-46.

19. Hanks GE, Pajak TF, Porter A, et al. Radiation Therapy Oncology Group. Phase III trial of long-term adjuvant androgen deprivation after neoadjuvant hormonal cytoreduction and radiotherapy in locally advanced carcinoma of the prostate: the Radiation Therapy Oncology Group Protocol 92-02. J Clin Oncol 2003; 21:3972-8.
20. Roach $M$, Lu JD, Lawton $C$, et al. A phase III trial comparing whole pelvic (WP) to prostata only (PO) radiotherapy and neoadjuvant to adjuvant total androgen suppression (TAS): preliminary analysis of RTOG 94-13 (abstract). Int J Radiat Oncol Biol Phys 2001; 51:3.

21. Lawton CA, Winter K, Murray K, et al. Updated results of the phase III Radiation Therapy Oncology Group (RTOG) trial 8531 evaluating the potential benefit of androgen suppression following standard radiation therapy for unfavorable prognosis carcinoma of the prostate. Int J Radiat Oncol Biol Phys 2001; 49(4):937-46.

22. Jani $A B$, Basu A, Abdalla I, Connell PP, Krauz L, Vijayakumar $S$. Impact of hormone therapy when combined with external beam radiotherapy for early-stage, intermediate-, or high-risk prostate cancer. Am J Clin Oncol. 2003; 26(4):382-5.

23. Nguyen KH, Horwitz EM, Hanlon AL, Uzzo R, Polack A. Does short-term androgen deprivation substitute for radiation dose in the treatment of high risk prostate cancer. Int J Radiat Oncol Biol Phys 2002; 54:2:133-5.

24. Zapatero A, Alcántara P, Valcárcel F, et al .Preliminary Report of a Multicenter Spanish Trial (GICOR 05) Of Risk-Adapted Androgen Ablation Combined With Dose-Escalation 3D Conformal Therapy For Prostate Cancer: Impact On Early Toxicity. Int J Radiat Oncol Biol Phys 2003; 57(Suppl 2). 